\title{
Two-Point LDV Measurements in a Plane Mixing Layer
}

\author{
M. M. Koochesfahani, ${ }^{*}$ C. J. Catherasoo, ${ }^{*}$ P. E. Dimotakis, $\dagger$ M. Gharib,${ }^{*}$ and D. B. Lang $\ddagger$ \\ California Institute of Technology, Pasadena, Calif.
}

\begin{abstract}
Investigations into the nature of the large structures in a two-dimensional shear layer were carried out using laser Doppler velocimetry in the GALCIT free-surface water tunnel. By simultaneous measurements of velocity at two points outside the turbulent region, above and below the shear layer, it was possible to measure the strength (total circulation) and location of the vorticity center of the large structures. It was found that structures not in the process of pairing convect downstream with the center of their cores close to the ray $y / x$ along which the mean velocity is given by $U_{m}=1 / 2\left(U_{1}+U_{2}\right)$. The determined value of the mean circulation is consistent with the independent measurements of the mean spacing between the structures. Results indicate that if the large structure vorticity distribution is elliptical, the inclination angle of its axis of symmetry with respect to the flow direction is small.
\end{abstract}

\section{Introduction}

$I^{\prime}$ $\mathrm{N}$ a recent experiment, ${ }^{1}$ the induced streamwise velocity fluctuations due to the passage of vortical structures in a two-dimensional shear layer were measured at a point just outside the mixing layer. Such a velocity record, however, (see Fig. 1), provides ambiguous information about the strengths and the core positions of the vortical structures, since the induced velocity due to the passage of a strong vortex far from the measuring station is indistinguishable from that due to a weaker vortex passing close to the measuring station.

To resolve this ambiguity, simultaneous measurements at two points were performed, above and below the shear layer, to allow the circulation (strength) and core position of the vortical structures to be determined.

\section{Apparatus, Instrumentation, and Data Processing}

A. Apparatus and the Resulting Flowfield

The flow apparatus and the resulting shear layer have been documented in Ref. 1. From the measured mean velocity profiles $\left(U_{2} / U_{1} \simeq 0.19\right)$, the vorticity (maximum slope) thickness $\delta_{\omega}$, normalized with the distance downstream from the splitter plate $x$, was found to be

$$
\delta_{\omega} / x \simeq 0.10
$$

The data presented in this paper were taken at $x=30 \mathrm{~cm}$ with $U_{1}=56 \mathrm{~cm} / \mathrm{s}$. These parameters yield a Reynolds number, defined by $\Delta U \delta_{\omega} / \nu$, of $1.4 \times 10^{4}$ at the measuring location, where $\Delta U$ is the velocity difference across the mixing layer. Note that the shear layer is fully turbulent at this Reynolds number.

\section{B. LDV Optics and Electronics}

The streamwise component of velocity was measured at the two points by a single-particle laser Doppler velocimeter (LDV) in the dual scatter mode. The two pairs of beams were

Received March 10, 1979; revision received July 3, 1979. Copyright (C) American Institute of Aeronautics and Astronautics, Inc., 1979. All rights reserved. Reprints of this article may be ordered from AIAA Special Publications, 1290 Avenue of the Americas, New York, N.Y. 10019. Order by Article No. at the top of page. Member price $\$ 2.00$ each, nonmember, $\$ 3.00$ each. Remittance must accompany order.

Index categories: Hydrodynamics; Jets, Wakes, and Viscid-Inviscid Flow Interactions.

*Graduate Student, Dept. of Aeronautics. Member AIAA.

$\dagger$ Assistant Professor, Aeronautics and Applied Physics. Member AIAA.

‡Graduate Student, Dept. of Applied Physics. generated by passing the laser beam through two cube beamsplitters at right angles (Fig. 2). The two focal volumes (measuring stations) were located at the high- and low-speed edges of the mixing layer, respectively (see Fig. 3).

The outputs of the two photodetectors were timemultiplexed at the input of a single LDV processor. The processor has been described in Ref. 1 .

\section{Data Processing}

The velocity data were low-pass filtered digitally according to the algorithm

$$
\begin{aligned}
\tilde{U}\left(t_{m}\right) & =\sum_{n} h\left(t_{m}-t_{n}\right) U\left(t_{n}\right) \delta t_{n} \\
\delta t_{n} & =t_{n}-t_{n-1}
\end{aligned}
$$

where $h$ is the (normalized) filter function, $U$ is the unfiltered, randomly sampled velocity data,

$$
\begin{aligned}
\tilde{U}\left(t_{m}\right) & =\sum_{n} h\left(t_{m}-t_{n}\right) U\left(t_{n}\right) \delta t_{n} \\
\delta t_{n} & =t_{n}-t_{n-1}
\end{aligned}
$$

where $h$ is the (normalized) filter function, $U$ is the unfiltered, randomly sampled velocity data, and $\tilde{U}$ is the filtered velocity data. The summation over $n$ is such that $-T_{f} \leq t_{m}-t_{n} \leq T_{f}$, where $2 T_{f}$ is the total width of the filter $h(t)$.

A Gaussian, low-pass filter was used. The filtered data were sampled uniformly (see Fig. 4).

\section{Data Reduction}

\section{A. Model}

Recent measurements ${ }^{1-6}$ have shown that the twodimensional shear layer is characterized by an array of large vortical structures, which are convected downstream with a velocity $U_{c}$, approximately given by $U_{c} \simeq 1 / 2\left(U_{1}+U_{2}\right)$. Now, in a frame moving with $U_{c}$, the induced velocities $u_{H}, u_{L}$, at the points $(x, b)$ and $(x,-b)$, respectively, when the center of vorticity of a particular structure is at $(x, c)$, are given by

$$
\begin{aligned}
& u_{H}=\frac{\Gamma}{2 \pi} \frac{61}{b-c} \\
& u_{L}=-\frac{\Gamma}{2 \pi} \frac{1}{b+c}
\end{aligned}
$$




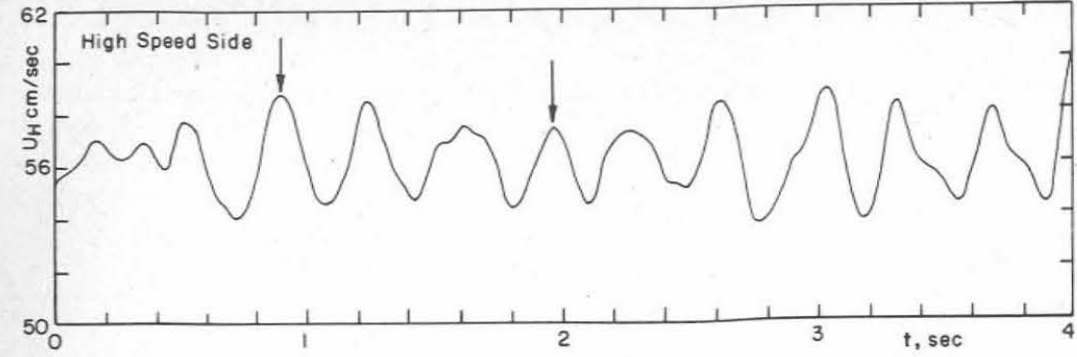

Fig. 1 Velocity record taken at a mean high-speed freestream velocity of $U_{1}=56 \mathrm{~cm} / \mathrm{s}$.

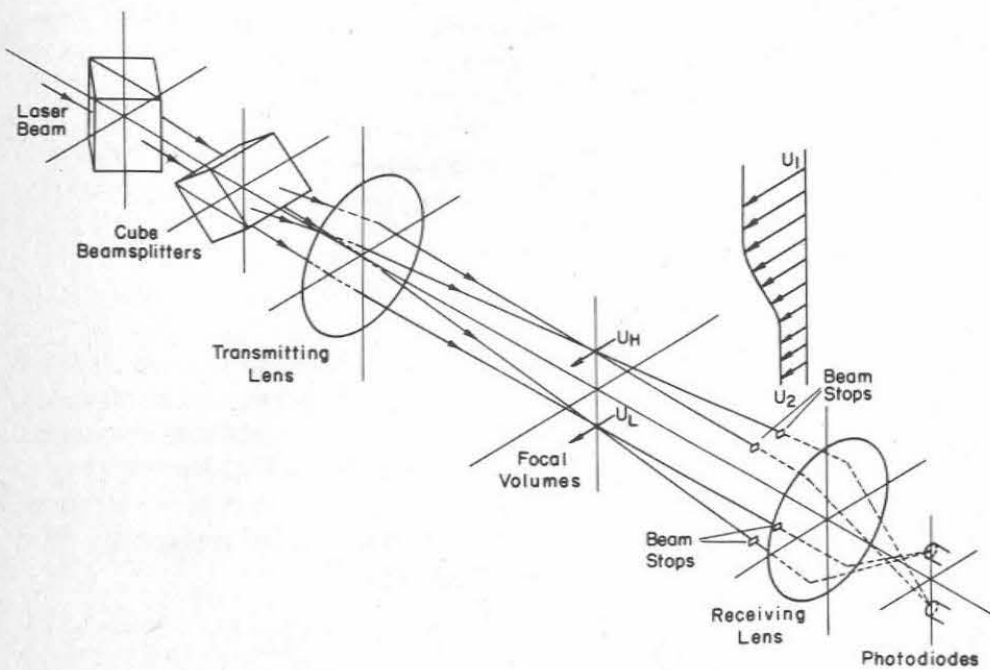

Fig. 2 LDV dual-scatter optical geometry (not to scale).

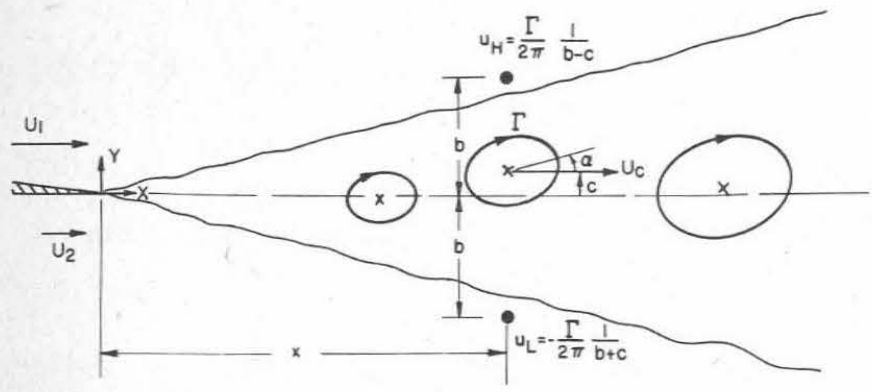

Fig. 3 Schematic diagram of the mixing layer. (The two filled dots denote the measurement locations.)

where $\Gamma$ is the total circulation of the vortical structure. Note that $\Gamma$ is defined to be positive for an induced velocity in the clockwise sense. Note also that the absolute values of the induced velocities reach a maximum at the instant the center of vorticity is on the line joining the measuring points. In this model, the effect of neighboring structures is neglected. It can be shown, however, that the resulting overestimation of the circulation $\Gamma$ is of the same order as the underestimation of $\Gamma$ caused by neglecting the expected higher moments (quadrupole moment) of the vorticity distribution. The effect of the quadrupole moment was estimated using the data in Fig. 6a of Ref. 2.

Using this model, an isolated, well-defined local maximum in the velocity measured on the high-speed side, associated with a corresponding well-defined local minimum on the lowspeed side, was interpreted as the signature of the passage of a single vortical structure not in the process of pairing. If additional significant disturbances were discernible in the maximum-minimum pair (interpreted as resulting from the presence of additional vortical structures), the pair was not included in the data. By way of example, only the data at $t=0.65,0.98,1.33,1.65,2.35,2.63$, and $3.35 \mathrm{~s}$ in Fig. 5 were considered.
The maximum induced velocity on the high-speed side and the corresponding minimum on the low-speed side were used in Eqs. (3) to compute the position of the vortex center $c$ and total circulation $\Gamma$ of each vortical structure. The results for 350 structures are presented in histogram form in Figs. 6 and 7.

\section{B. Cross Correlation and Autocorrelation Functions}

The correlation functions of $u_{H}^{\prime}=u_{H}-\bar{u}_{H}$ and $u_{L}^{\prime}=u_{L}-$ $\bar{u}_{L}$, the velocity fluctuations on the high- and low-speed sides, respectively, were computed from the filtered data by means of the equations

$$
\begin{aligned}
& R_{H L}(\tau)=\frac{1}{M} \sum_{m=1}^{M} u_{H}^{\prime}\left(t_{m}\right) u_{L}^{\prime}\left(t_{m}+\tau\right) \\
& R_{H H}(\tau)=\frac{1}{M} \sum_{m=1}^{M} u_{H}^{\prime}\left(t_{m}\right) u_{H}^{\prime}\left(t_{m}+\tau\right) \\
& R_{L L}(\tau)=\frac{1}{M} \sum_{m=1}^{M} u_{L}^{\prime}\left(t_{m}\right) u_{L}^{\prime}\left(t_{m}+\tau\right)
\end{aligned}
$$

where, for each time shift $\tau, M$ is the largest index, such that $t_{M}+\tau \leq T, T$ being the record length. The results, averaged over 30 records, are plotted in Figs. 8 and 9. The smooth curves through the data points have been drawn to aid the eye.

\section{Results and Discussion}

The mean core position (Fig. 6) is slightly below (i.e., on the low-speed side) the ray $c=0$, on which $U_{m}=1 / 2\left(U_{1}+U_{2}\right)$. The small dispersion in the histogram indicates that structures not in the process of pairing convect downstream with their cores closely spaced about a single ray. 
Fig. 4 Raw and filtered velocity data samples.
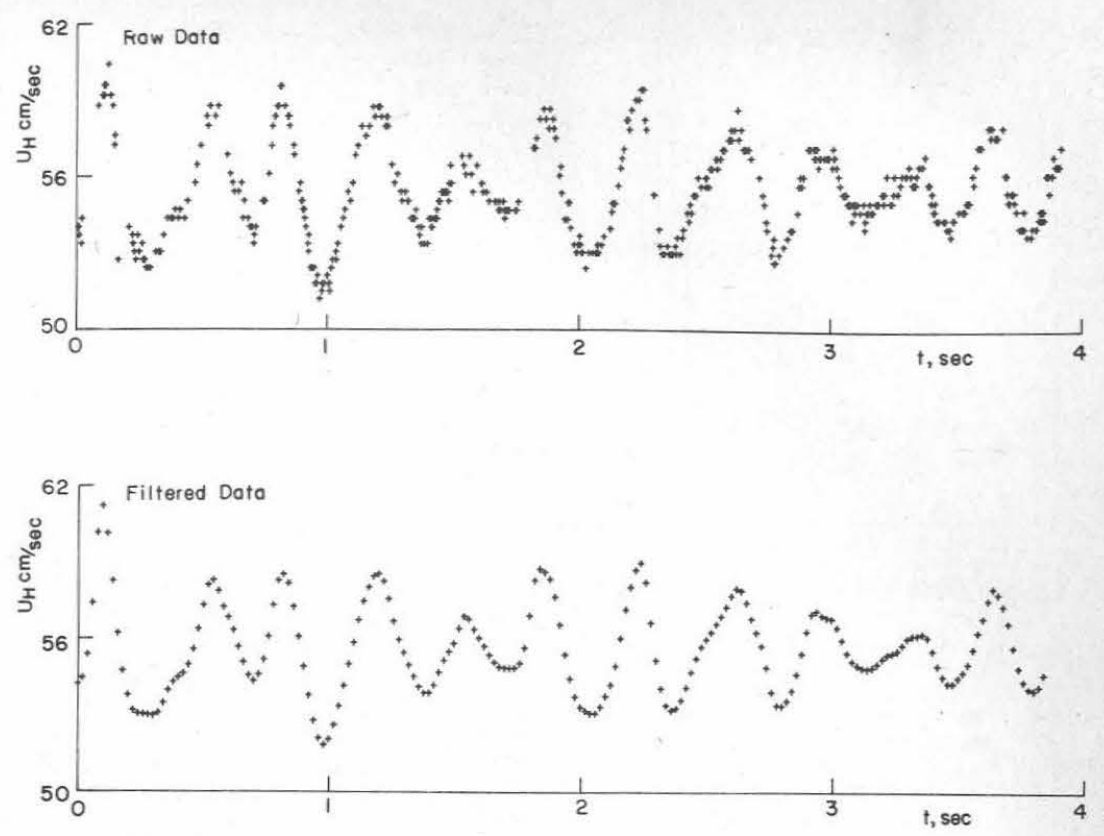

Fig. 5 Simultaneous velocity records at the high- and low-speed edges of the shear layer.

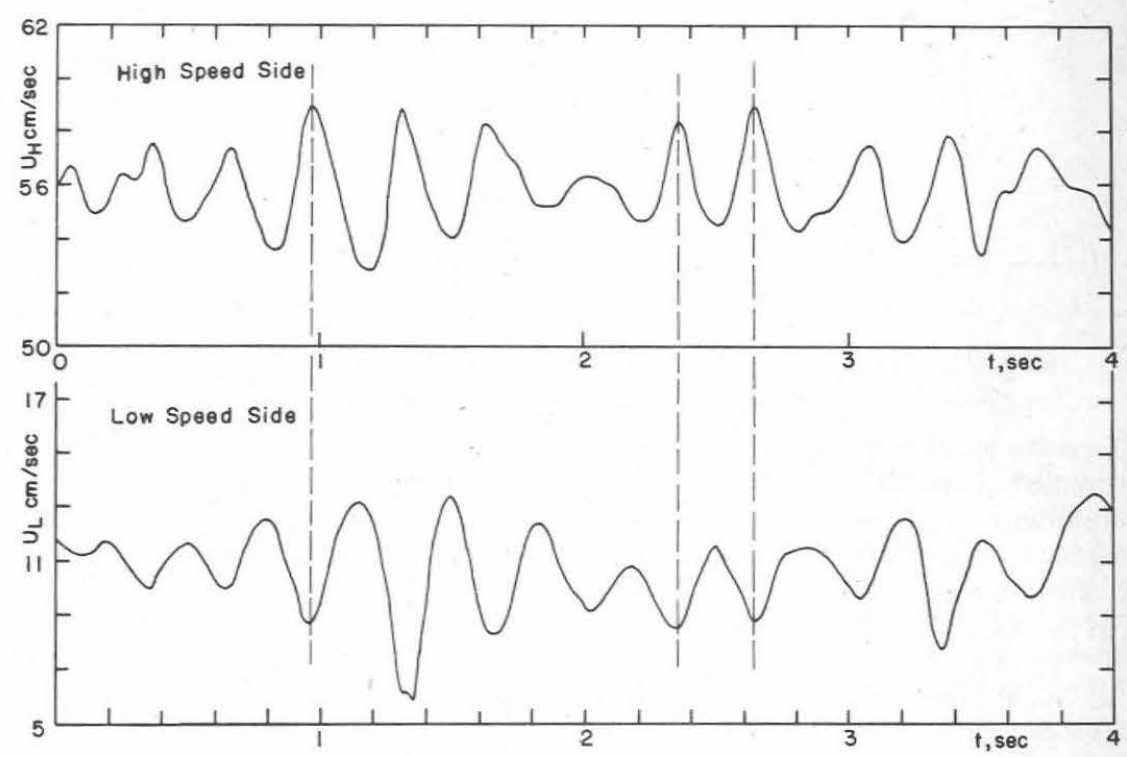

The mean circulation $\bar{\Gamma}$ (Fig. 7), normalized by the product $\delta_{\omega} \Delta U$, is given by

$$
\bar{\Gamma} / \delta_{\omega} \Delta U \simeq 3.9
$$

where $\Delta U=U_{1}-U_{2}$ is the velocity difference across the shear layer. Note that from the boundary condition

$$
\bar{\Gamma} / \ell \simeq \Delta U
$$

and from Eq. (5) we estimate $\bar{\ell}$, the spacing between structures, to be

$$
\bar{\ell} \delta_{\omega} \simeq 3.9
$$

This result is consistent with earlier independent measurements of this ratio. ${ }^{1,4}$ It should be mentioned that, strictly speaking, the boundary condition given by Eq. (6) should be written instead as $(\overline{\Gamma / \ell})=\Delta U$. The use of the ratio of the mean quantities is justified a posteriori in this case, since the distribution of $\Gamma$ is narrowly peaked about its mean value (see Fig. 7).

\section{Crosscorrelation and Autocorrelation Results}

Results of other investigators $2,4,5$ suggest that the vortical structures in a mixing layer are, by and large, cylinders of elliptical cross section whose major axes may be inclined with respect to the streamwise direction (see Fig. 3). If the angle of inclination is positive, the velocity on the high-speed side will reach its maximum before the low-speed side velocity reaches its minimum. This would cause the minimum of the crosscorrelation function of the high- and low-speed velocity fluctuations to occur at the positive time shift. It should be noted, however, that the minimum of the crosscorrelation function has occurred at a negative time shift (see Fig. 9). We believe that pairing is responsible for this result.

In pairing, neighboring vortices rotate around each other and coalesce to form a larger one. Using Fig. 3 of Ref. 5, a schematic diagram of this process and the resulting induced velocity fluctuations are sketched on Fig. 10. It can be seen that, in the process of pairing, a pair of vortices taken together can be assigned an effective negative angle of inclination.

The pairing process also affects the autocorrelation functions of the velocities on each side of the mixing layer (Fig. 8). 


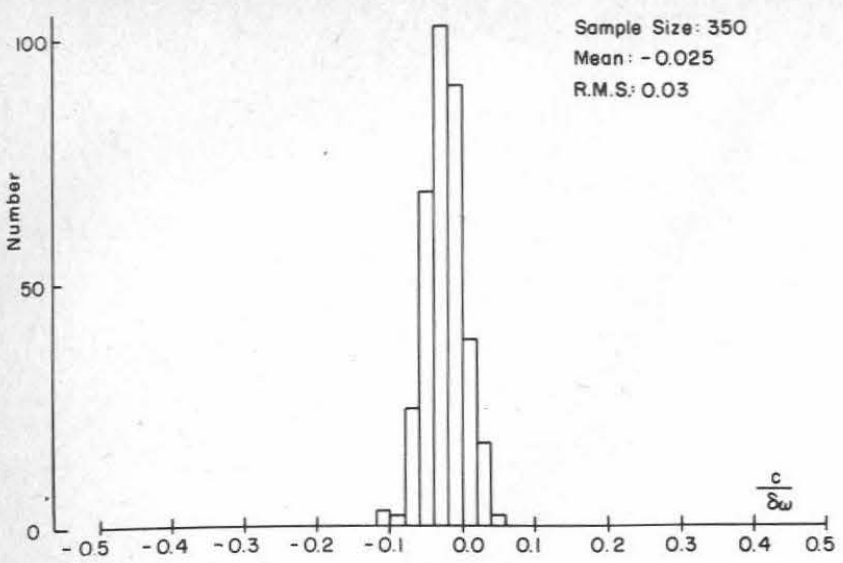

Fig. 6 Distribution of normalized vortex core offset distances.

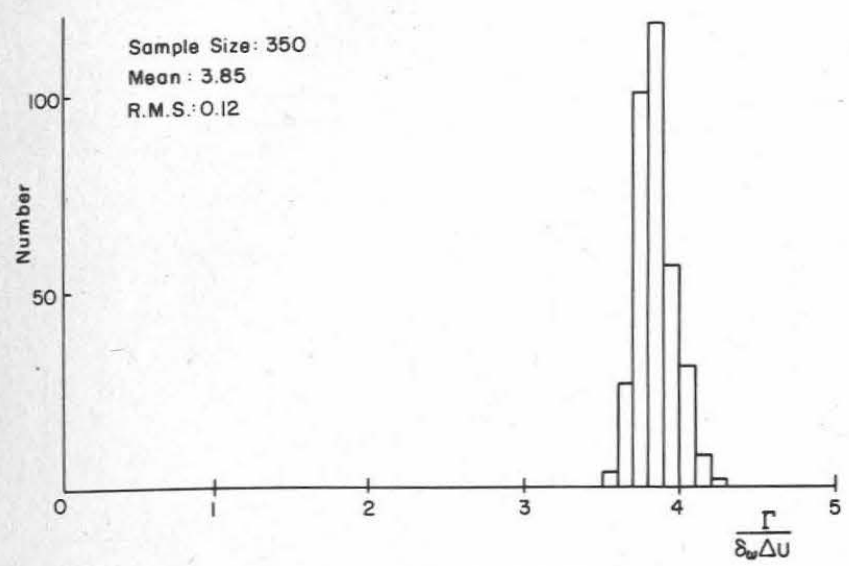

Fig. 7 Distribution of normalized vortex strengths.

The autocorrelation function of the high-speed side data gives a smaller characteristic time, $\tau_{H}$, (twice the time to the first minimum) than that for the low-speed side (i.e., $\tau_{L}$ ). This becomes clear when one considers the fact that, in the process of pairing, the vortex on the high-speed side must be accelerating, while the vortex on the low-speed side is decelerating. Note that this is consistent with the results of Ref. 7. It should also be noted that the data support the relation

$$
\tau_{H L} \simeq 1 / 2\left(\tau_{H}+\tau_{L}\right)
$$

where $\tau_{H L}$ is the characteristic time determined from the crosscorrelation function (twice the time interval between the minimum and maximum).

If we use the characteristic time $\tau_{H L}$ derived from the crosscorrelation function as a measure of the mean time between the passage of the large structures, the mean spacing would then be given by

$$
\bar{\ell} \simeq \tau_{H L} U_{c}
$$

where, from the crosscorrelation function, we have

$$
\tau_{H L} U_{c} / x=0.40
$$

Combining Eqs. (1, 9, and 10) yields

$$
\overline{\ell / \delta_{\omega}} \simeq 4.0
$$

Note that the independent estimates of $\overline{\ell / \delta} \delta_{\omega}$, as given by Eqs. (7) and (11), are consistent.

Direct examination of the velocity records shows that the maximum velocity on the high-speed side and the minimum

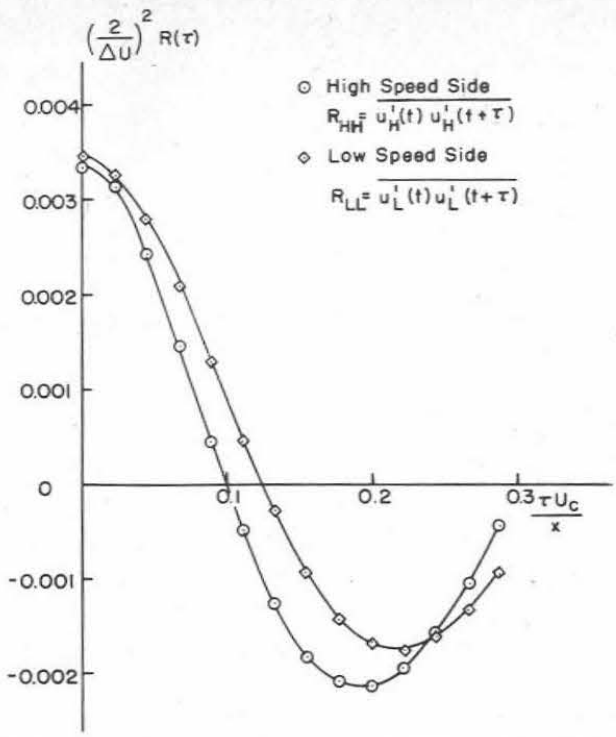

Fig. 8 Autocorrelation functions of velocities on the high- and lowspeed sides.

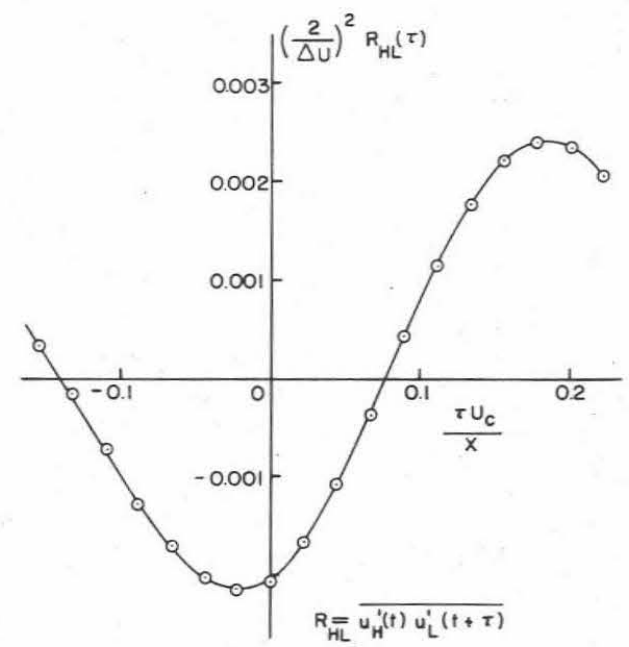

Fig. 9 Cross-correlation function of high- and low-speed velocities.
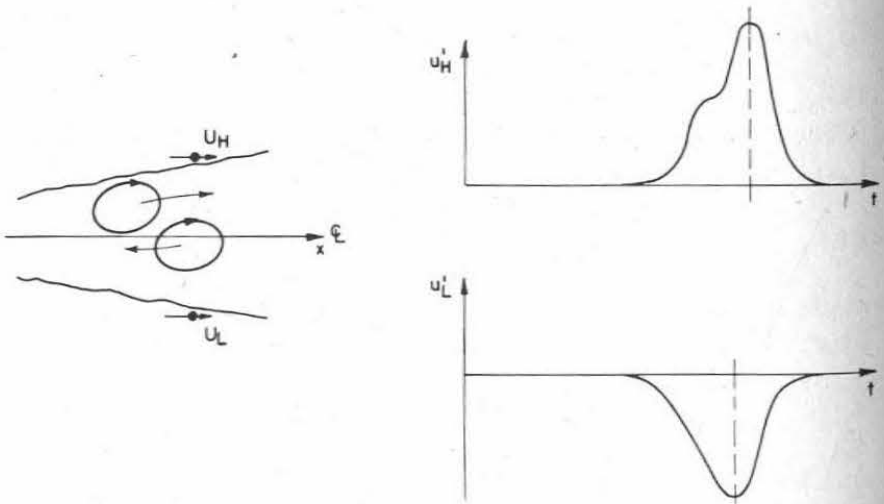

Fig. 10 Schematic diagram of two vortices in the process of pairing and the resulting induced velocity fluctuations.

velocity of the low-speed side, corresponding to the passage of a single structure, occur at the same time, within the accuracy of the present measurements. Note the time markers on Fig. 5. This indicates that if the vorticity distribution is elliptical, the inclination angle is small. This is consistent with the findings of the stability analysis by Moore and Saffman. ${ }^{8}$ 


\section{Conclusion}

Simultaneous records of the streamwise component of velocity at the high- and low-speed edges of the mixing layer were used to calculate the core position and strengths (circulation) of the vortical structures. It was found that an isolated structure (not in the process of pairing) travels with the center of its core very close to the ray $y / x$ on which the mean velocity is $U_{m}=1 / 2\left(U_{1}+U_{2}\right)$ and has a mean circulation given by $\bar{\Gamma} / \delta_{\omega} \Delta U \simeq 3.9$. The small dispersion about the mean of the distributions of core position and circulation is noteworthy.

\section{Acknowledgment}

The measurements were conducted under the sponsorship of the Experimental Methods course of the Graduate Aeronautical Laboratories at Caltech and the Air Force Office of Scientific Research Contract No. F44620-76-C0046. D. Lang would like to acknowledge the support of the Fannie and John Hertz Foundation.

\section{References}

${ }^{1}$ Dimotakis, P. E. and Brown, G. L., "The Mixing Layer at High Reynolds Number: Large Structure Dynamics and Entrainment,"
Journal of Fluid Mechanics, Vol. 78, No. 3, 1976, pp. 535-560+2 plates.

${ }^{2}$ Browand, F. K. and Weidman, P. E., "Large Scales in the Developing Mixing Layer," Journal of Fluid Mechanics, Vol. 76, No. 1, 1976, pp. 127-144.

${ }^{3}$ Brown, G. and Roshko, A., "The Effect of Density Differences on the Turbulent Mixing Layer," Turbulent Shear Flows, AGARDCP-93, Paper 23, 1971.

${ }^{4}$ Brown, G. L. and Roshko, A., "'On Density Effects and Large Structure in Turbulent Mixing Layers," Journal of Fluid Mechanics, Vol. 64, 1974, pp. 775-816.

${ }^{5}$ Roshko, A., "Structure of Turbulent Shear Flows: A New Look," AIAA Paper 76-78; also AIAA Journal, Vol. 14, 1976, pp. 1348-1357 and Vol. 15, 1976, p. 768.

${ }^{6}$ Winant, C. D. and Browand, F. K., "Vortex Pairing: The Mechanism of Turbulent Mixing-Layer Growth at Moderate Reynolds Number,"' Journal of Fluid Mechanics, Vol. 63, No. 2, 1974, pp. 232255 .

${ }^{7}$ Oster, D., "An Experimental Investigation of the TwoDimensional Turbulent Mixing Layer," M.Sc. Thesis, Tel-Aviv University, Israel, July 1976.

${ }^{8}$ Moore, D. W. and Saffman, P. G., "The Density of Organized Vortices in a Turbulent Mixing Layer," Journal of Fluid Mechanics, Vol. 69 , No. 3, 1975, pp. 465-473.

\title{
From the AIAA Progress in Astronautics and Aeronautics Series...
}

\section{INTERIOR BALLISTICS OF GUNS-v. 66}

\author{
Edited by Herman Krier, University of Illinois at Urbana-Champaign, \\ and Martin Summerfield, New York University
}

In planning this new volume of the Series, the volume editors were motivated by the realization that, although the science of interior ballistics has advanced markedly in the past three decades and especially in the decade since 1970, there exists no systematic textbook or monograph today that covers the new and important developments. This volume, composed entirely of chapters written specially to fill this gap by authors invited for their particular expert knowledge, was therefore planned in part as a textbook, with systematic coverage of the field as seen by the editors.

Three new factors have entered ballistic theory during the past decade, each it so happened from a stream of science not directly related to interior ballistics. First and foremost was the detailed treatment of the combustion phase of the ballistic cycle, including the details of localized ignition and flame spreading, a method of analysis drawn largely from rocket propulsion theory. The second was the formulation of the dynamical fluid-flow equations in two-phase flow form with appropriate relations for the interactions of the two phases. The third is what made it possible to incorporate the first two factors, namely, the use of advanced computers to solve the partial differential equations describing the nonsteady twophase burning fluid-flow system.

The book is not restricted to theoretical developments alone. Attention is given to many of today's practical questions, particularly as those questions are illuminated by the newly developed theoretical methods. It will be seen in several of the articles that many pathologies of interior ballistics, hitherto called practical problems and relegated to empirical description and treatment, are yielding to theoretical analysis by means of the newer methods of interior ballistics. In this way, the book constitutes a combined treatment of theroy and practice. It is the belief of the editors that applied scientists in many fields will find material of interest in this volume. 